The propositions of the first paragraph above are special cases of the Corollary. As has been pointed out by Pearcy and Shields [5], it is not known if there are any operators $A$ which do not satisfy the hypotheses of the consequence of Lomonosov's work; (Cowen [1] has recently shown that the unilateral shift commutes with an operator which commutes with a compact operator). Thus we certainly do not know of any operators which fail to satisfy the more general criteria following from [2].

Added in pro of. There are operators that do not satisfy Lomonosov's hypotheses; see the paper by Haduin, E. Nordgren, H. Radjavi and P. Rosenthal in J. Funct. Anal. 38 (1980), $410-415$.

\title{
References
}

[1] Carl C. Cowe n, An analytic Toeplitz operator that commutes with a compact operator, J. Funct. Anal. 36 (1980), 169-184.

[2] C. K. Fong, E. Nordgren, M. Radjabalipour, H. Radjavi and P. Ros e $\mathrm{n}$ h a l, Extensions of Lomonosov's invariant subspace theorem, Acta Sci. Math. (Szeged) 41 (1979), 55-62.

[3] V. I. L o m o n o s ov, Invariant subspaces for the family of operators which commute with a completely continuous operator, Funct. Anal. and Appl. 7 (1973), 213-214.

[4] E. Nordgren, M. Radjabalipour, H. Radjavi and P. Rosentha 1 Algebras intertwining compact operators, Acta. Sci. Math. (Szeged) 39 (1977), 115-119.

[5] C. P e r cy and A. L. S hi eld s, A survey of the Lomonosov technique in the theory of invariant subspaces, pp. 220-229 of Topics in Operator Theory, Amer. Math. Soc. Surveys No. 13, Providence R.I., 1974.

[6] Heydar Radjavi and Peter Rosent ha 1, Invariant Subspaces, Springer-Verlag, BerlinHeidelberg-New York 1973.

Presented to the semester

$$
\text { Spectral Theory }
$$

September 23-December 16, 1977
BANACH CENTER PUBLICATIONS, VOLUME 8

PWN-POLISH SCIENTIFIC PUBLISHERS

\section{SOME QUESTIONS IN OPERATOR THEORY AND APPLICATIONS} IN ANALYSIS

\section{JAMES ROVNYAK}

Department of Mathematics, University of Virginia, Charlottesville, Virginia, USA

The questions fall into two areas. In both cases, full accounts will appear elsewhere in [1], [2].

\section{Invariant subspaces of finite convolution operators}

A finite convolution operator is an operator on $L^{2}(0,1)$ having the form

$$
T: f(x) \rightarrow \int_{0}^{x} k(x-t) f(t) d t,
$$

where $k \in L^{1}(0,1)$. By-a symbol for such an operator we mean any function of the form

$$
A(z)=\int_{0}^{1} e^{i t z} k(t) d t+e^{i z} G(z),
$$

where $G(z)$ is analytic and bounded in some half-plane $y>\eta$, where $\eta$ is a real number. A survey is made of recent progress on the problems of giving conditions on symbols for the unicellularity and similarity of such operators, and, more generally, of arbitrary operators in the commutant of the integration operator

$$
J: f(x) \rightarrow \int_{0}^{x} f(t) d t
$$

on $L^{2}(0,1)$. A table of known examples is given. A section of the paper lists open problems in the theory.

\section{Cayley inner functions and applications in analysis}

A Cayley inner function is defined to be any analytic function $\xi(z)$ satisfying $\xi(z)$ $=\xi\left(z^{*}\right)^{*}$ for $z \neq z^{*}$ (if $z=x+i y$, then $z^{*}=x-i y$ ), $\operatorname{Im} \xi(z)>0$ for $\operatorname{Im} z>0$, such that $\xi(x)=\xi(x+i 0)$ is real a.e. on the real-axis. We say that $\xi$ maps a real 
Borel set $\Delta$ onto an interval $(c, d)$ of the real line if $\xi(x) \in(c, d)$ for almost all $x \in \Delta$, and $\xi(x) \notin(c, d)$ for almost all $x \in R \backslash \Delta$. Then

$$
\int_{\Delta} \frac{z-w^{*}}{(t-z)\left(t-w^{*}\right)} f(\xi(t)) d t=\int_{c}^{d} \frac{\xi(z)-\xi(w)^{*}}{(t-\xi(z))\left(t-\xi(w)^{*}\right)} f(t) d t
$$

for all nonreal numbers $z$ and $w$ and any function $f(x)$ such that $\left(1+x^{2}\right)^{-1} f(x)$ $\in L^{1}(c, d)$. If also

$$
\lim _{y \rightarrow \infty} \xi(i y) /(i y)=\beta>0,
$$

then for any $f(x) \in L_{p}(c, d), 1 \leqslant p<\infty$, and any nonreal number $z$,

$$
\int_{\Delta} \frac{f(\xi(t))}{t-z} d t=\int_{c}^{d} \frac{f(t)}{t-\xi(z)} d t
$$

and

$$
\int_{\Delta}|f(\xi(t))|^{p} d t=\beta^{-1} \int_{c}^{d}|f(t)|^{p} d t
$$

furthermore, in case $p=1$,

$$
\int_{\Delta} f(\xi(t)) d t=\beta^{-1} \int_{c}^{d} f(t) d t
$$

Under the stated assumptions, the integrals on the left sides of (1)-(4) converge absolutely. Analogous formulas are proved for singular integrals. Applications are given to approximation theory, distribution function formulas for Hilbert transforms, and orthogonal expansions and isometric operators in $L^{2}$ spaces.

\section{References}

[1] R. Fra $\mathrm{nkfurt}$ and J. R $\mathrm{v} \mathrm{n}$ y a $\mathrm{k}$, Recent results land unsolved problems on finite convolution operators, Linear Spaces and Approximation, ed. P. L. Butzer and B. Sz.-Nagy, pp. 133-150, Birkhäuser Verlag, Basel 1978.

[2] M. Ros en blum and J. R o v y a k, Change of variables formulas with Cayley inner functions, Topics in Fumftional Analysis, Adv. in Math. Supplementary Studies, vol. 3, pp. 283-320, Academic Press, New York 1978.

$$
\begin{aligned}
& \text { Presented to the semester } \\
& \text { Spectral Theory } \\
& \text { September 23-December 16, } 1977
\end{aligned}
$$

\section{A NOTE ON SEMICHARACTERS}

\author{
Z. SŁODKOWSKI and W. ŻELAZKO
}

Institute of Mathematics of the Polish Academy of Sciences, Warszawa, Poland

We introduce here a concept of semicharacter of a complex Banach algebra. We show that the algebra of all $n \times n$ matrices with complex entries possesses a proper semicharacter if and only if $n=2$. We prove the absence of semicharacters for algebras $B(X)$ of all bounded endomorphisms of some classical Banach spaces. We also investigate relations between semicharacters and minimal subspectra and, finally, we give the definition of a semicharacter of a locally compact group.

\section{Semicharacters of Banach algebras}

Definition 1.1. Let $A$ be a complex Banach algebra. A semicharacter on $A$ is a complex-valued function $\varphi$ defined on $A$ such that for every commutative subalgebra $\mathscr{A} \subset A$ the restriction of $\varphi$ to $\mathscr{A}$ is a multiplicative-linear functional (= a character) on $\mathscr{A}$. We do not assume that $\varphi$ is a continuous function. In case when $A$ possesses the unit element $e$, we assume also that $\varphi$ is not identically equal to zero, i.e. $\varphi(e)=1$.

If $A$ has no unit element and $A_{1}=A \oplus\{C e\}$ is its unital extension, then every semicharacter $\varphi$ on $A$ extends to a semicharacter $\varphi_{1}$ on $A_{1}$ given by $\varphi_{1}(x+\lambda e)$ $=\varphi(x)+\lambda, x \in A, \lambda \in C$.

Let us also remark that if a semicharacter is a linear functional on $A$, then it is multiplicative and linear, i.e. a character on $A$ (cf. [1]). And so, we say that a semicharacter is proper if it is not a character, i.e. if it is not a linear functional.

We shall now describe all semicharacters of the algebras of all linear endomorphisms of $n$-dimensional Euclidean spaces, $n=2,3, \ldots$ (for $n=1$ it is the algebra isomorphic to the field $C$ and it has a character - the identity map onto itself). Since all these algebras are simple, then all possible semicharacters are proper. If $X$ is a Banach space, $B(X)$ will stand for the Banach algebra of all bounded endomorphisms of $X$. With this notation we have the following

THEOREM 1.2. Let $A=B\left(C^{n}\right), n=1,2, \ldots$ Then $A$ possesses $2^{c}$ (proper) semicharacters if $n=2$ and no semicharacters if $n \geqslant 3$. Here $c$ is the cardinality of continuum. 